\title{
A Procedure for Performance Experimental Analysis of a Globe Control Valve
}

\author{
Hesam Hoursan \\ Ph.D Student, School of Mechanical Engineering \\ Sharif University of Technology \\ Researcher at Rasta Group Industrial Valve Co. \\ Tehran, Iran \\ Hesam.hoursan74@ student.sharif.edu
}

\author{
Mohammad Omid Hadjiazim \\ Graduate, Civil Engineering \\ University of Tehran \\ Researcher at Rasta Group Industrial Valve Co. \\ Tehran, Iran \\ Omid.azimi@rastagroup.net
}

\author{
Mohammad J. Moradi \\ M.S. Graduate, Petroleum Engineering Department, \\ Amirkabir University of Tech \\ Researcher at Rasta Group Industrial Valve Co. \\ Tehran, Iran \\ Mohamad.moradi89@yahoo.com
}

\author{
Mohammad Taghi Ahmadian \\ Professor, School of Mechanical Engineering \\ Sharif University of Technology \\ Tehran, Iran \\ Ahmadian@sharif.edu
}

\begin{abstract}
Ahmad Barari
Faculty of Engineering and Applied Science

University of Ontario Institute of Technology

Oshawa, Ontario, Canada

Ahmad.barari@uoit.ca
\end{abstract}

\begin{abstract}
Control valves are known as the final control element in hydraulic closed/open loops of modern process industries around the world. Proper selection of control valve leads to enhanced performance curve of the hydraulic systems and therefore increases the efficiency, reliability, profitability and safety of the system. Flow coefficient (CV) of a control valve describes the relation between the pressure drop across the valve and the flow passing through it. Despite many computational efforts for calculating the exact value and curve of $\mathrm{CV}$, the experimental procedure of the $\mathrm{CV}$ test has not been documented well. We used a control valve test-set up designed based on the standards ANSI/ISA-75.02-1996 and IEC 605342-3 (2013) to evaluate the performance of a 3 in. control valve. Upon extracting the results in terms of inlet, and outlet pressure and flow, the characteristic parameters such as CV and opening percentage were derived and compared with an ideal curve. Error analysis was performed to account for the tolerance of the measured parameters by the measuring devices. The results show acceptable agreement within the criteria of a reference standard approving the validity of the design method.
\end{abstract}

Keywords- Control valve; Flow coefficient, Ideal curve, Test setup, Performance

\section{1- INTRODUCTION}

Control valves have long been used as the final control elements in various types of process lines. They are typically known to be in charge of one-third of the total pressure drop along the hydraulic line which represents a high impact on the regulation of total line performance curve. The coefficient of valve $(\mathrm{CV})$ is related to the surface area through which the fluid can throttle (pass). Such area is controlled via moving (linear) or rotating (rotary) a closure element relative to a fixed housing (seat). The position of the closure element is controlled by various types of actuators including pneumatic, electric, electrohydraulic and hydraulic actuators; among which pneumatic actuators are simpler and more common.

A Globe valve with linear actuator is composed of body, bonnet, plug, seat, stem, and for the case where more pressure drop is required at the price of less $\mathrm{CV}$, various types of cage are used. Flow coefficient $(\mathrm{CV})$ of a control valve describes the relation between the pressure drop across the valve and the flow passing through it. The definition of Control Valve Coefficient was soon accepted and applied universally after its first introduction to industry by Masoneilan in 1944. [1] In more practical terms, the flow coefficient CV is the volume (in US gallons) of water at $60^{\circ} \mathrm{F}$ that will flow per minute through a 
valve with a pressure drop of 1 psi across the valve. [2, 3] A comprehensive laboratorial set-up is required for fine measurement of variables such as flow and pressure drop, from which the CV can be calculated and plotted. The specifications of such set-up for incompressible flow measurement are mentioned in ANSI/ISA-75.02-1996 and IEC 60534-2-3 (2013) standards $[4,5]$. Previous researchers have implemented the testing process such as that of Vikas et al., in which a 4 inch globe control valve is tested experimentally for its equal percentage and linear performance curves. [6] Computational methods have been presented by Guy Borden [7] to calculate and plot the ideal performance characteristics for throttling valves which include linear and equal percentage characteristics of the valve. Also, Aragon et al. have presented a method for experimental determination of valve capacity with compressible flow [8].

Despite the achievements of previous publications, a full procedure for testing and analysis of a control valve performance curve has not been proposed. Since such analysis can serve as a validation method for the control valve design and on-site performance, it is of critical importance to consider the effects of various variables such as hysteresis in sequential opening and closing of the valve, error analysis and considering the existing criteria [9] for acceptance of the curve, on which few documents have been published. The current study aims to analyze the results of a performance test on a 3 inch commercial Globe Control Valve with equal percentage trim to represent a nonlinear characteristic behavior.

\section{2- METHODOLOGY}

\section{2-1- Valve Characteristics}

A 3 inch. globe control valve manufactured with modern technology was tested. The specifications of the test valve are shown in Table 1.

The trim of the top guided valve is a contoured plug head with equal percentage characteristics. The contoured plug head of the valve is designed based on the computations of [7] for a $3 * 2.5$ in valve with $40 \mathrm{~mm}$ of stroke. A computational code in MATLAB was used to estimate the curve of the contoured plug (Figure 1). The control valve is equipped with a single acting pneumatic actuator and a Yamatake positioner with $0.1 \%$ travel accuracy for fine positioning of the stem during opening and closing cycles.
Table 1- Type test characteristics and dimensions

\begin{tabular}{|l|c|}
\hline Valve Type \& Rating & Globe Top Guided GS1000- ANSI 300 \\
\hline Valve Size & 3 in. Manifold *2.5 in Plug \\
\hline Valve Rated CV (Fully Open) & 72 \\
\hline Valve Characteristic & $\begin{array}{c}\text { Equal percentage with 0.05 of Rated CV } \\
\text { at Min. }\end{array}$ \\
\hline Actuator Type & \begin{tabular}{c} 
Pneumatic Single Act. \\
\hline Positioning
\end{tabular} \\
\hline
\end{tabular}
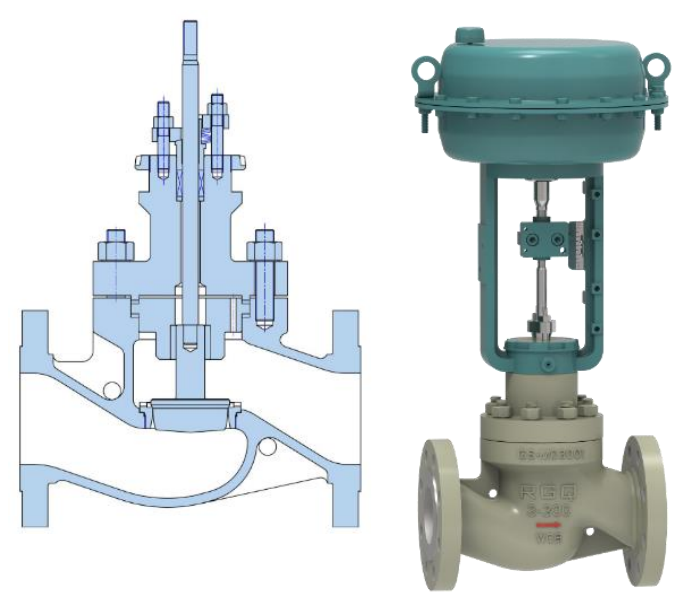

Figure 1- Top Guided Globe Valve Trim Style (Courtesy of Rasta Group Industrial Valve Co.)
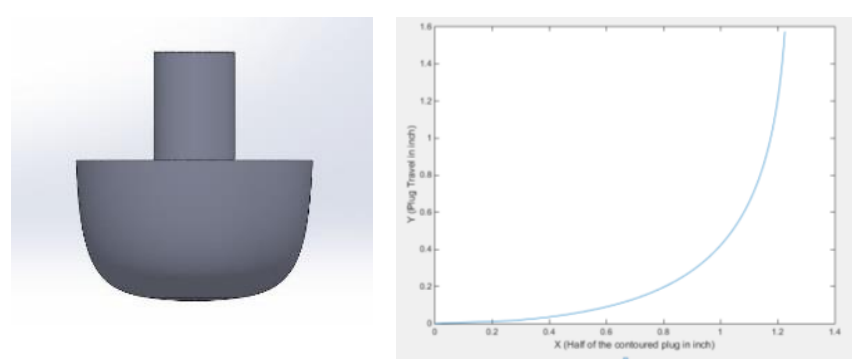

Figure 2- Left: Plug head curve (half of the contoured plug) designed for the $3 * 2.5$ in valve with $40 \mathrm{~mm}$ stroke and recovery factor of 0.9 Right: a MATLAB code is written for computation of the curve based on [7] (courtesy of Rasta Group Industrial valve Co.)

\section{2-2- Laboratory Test Set-up}

A laboratorial test set-up which has been designed for 3 in. valves was prepared for the study. (Figure 4) The set-up was verified for dimensional agreement with ANSI/ISA 75.021996. [9] (Figure 3). The standard ANSI/ISA-75.01.01-2002 (2002) was used for sizing calculations which defines equations 
for sizing control valves and showing the result as a flow capacity coefficient, CV. $[1,2]$

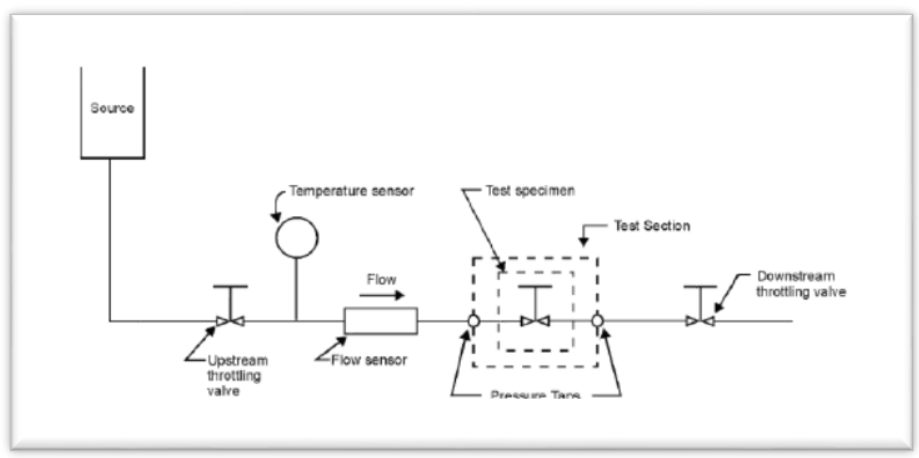

Figure 3- Test Circuit Suggested by ANSI/ASME 75.02 for testing the performance characteristic of control valves $[3,4]$

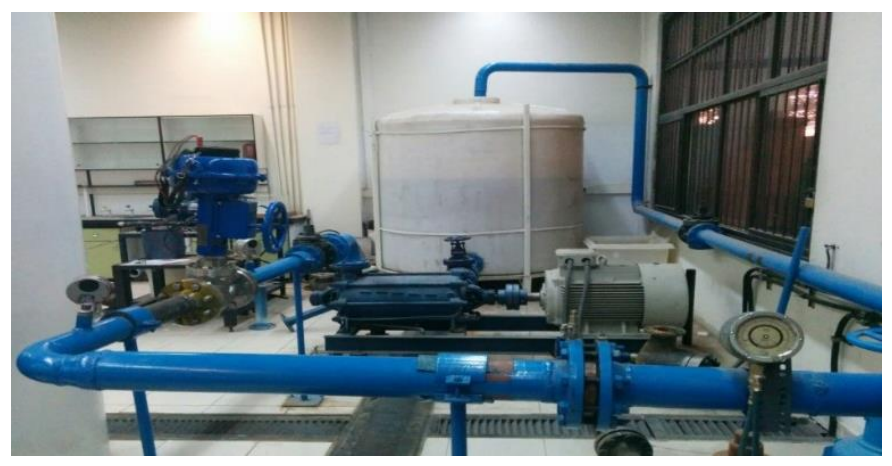

Figure 4- The test bench used for the experiment (Courtesy of Thermohydraulic Laboratory of the Iranian Ministry of Power)

The test bench instrumentation was chosen to meet the specifications of standards ANSI/ISA-75.02-1996 (1996) and ANSI/ISA-75.01.01-2002 (2002). [4, 5] Three physical variables were obtained at each run: pressure, temperature and flow rate, with the following characteristics,

Pressure: All pressure measurements are made with an error not exceeding $\pm 2 \%$ of the actual value. [4, 5] Rosemount 3051 Pressure Transmitters are used to meet the specified accuracy. (Figure 5)

Temperature: The flow temperature should be measured within an error not exceeding $\pm 1^{\circ} \mathrm{C}\left( \pm 2^{\circ} \mathrm{F}\right)$ of actual value. [4, 5] A standard temperature sensor is used to make sure the temperature of water is within acceptable limits.

Flow: The flow rate instrumentation may be any device that meets specified accuracy. This instrument is used to determine the true time average flow rate within an error not exceeding \pm $2 \%$ of the actual value. $[4,5]$ The resolution and repeatability of the instrument must be within $\pm 0.5 \%$. A Magnetic MAXIFLO flow-meter with the accuracy of $2 \%$ has been used to measure the flow. Three readings are made each time and the average is calculated and reported as data.

Pump: A centrifugal Pump with maximum flow capacity of $120 \mathrm{~m} 3 / \mathrm{h}$ and suitable pressure limit for 4 in piping is used.
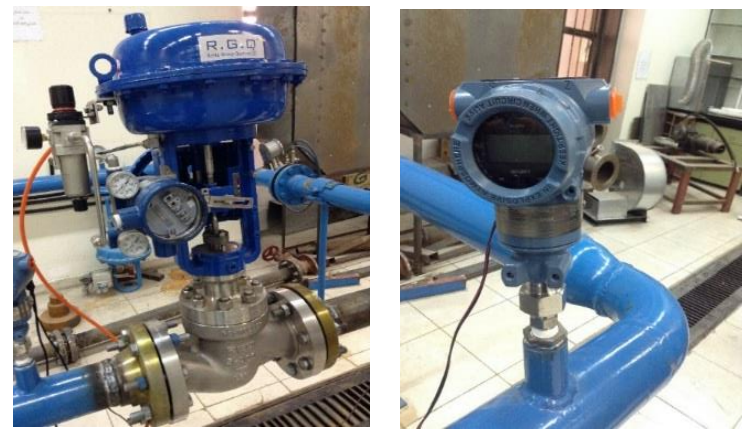

Figure 5- Valve installed in 3-in pipeline (left), Pressure transducers installed on pressure taps (right)

\section{2-3- Test procedure}

The set-up was used to test the valve through stepwise closing and opening of the valve and recording the measured parameters including differential pressure across the valve and flow. The test was repeated in three cycles of closing-openingclosing of the valve and during the steps, data was recorded on every $6.25 \%$ change in stem travel (This is equivalent to $1 \mathrm{~mA}$ change in positioner signal input in the range of 4-20 mA current).

Scenario A- Closing the valve from fully-open condition (maximum $\mathrm{Cv}$ ) to minimum flow at 0.05 of maximum $\mathrm{Cv}$. And recording data including: 1 - Inlet and Outlet static pressure using digital pressure gauges. 2- Flow passing through valve measured by a magnetic flow-meter. Results were plotted as calculated $\mathrm{Cv}$ vs. travel percentage to characterize the valve. Attempt was made to avoid choking conditions which correspond to maximum allowable pressure drop.

Scenario B- The above step was repeated from fully-closed condition to fully open.

Scenario C- The above step was repeated once more from fully-open condition to fully-closed.

Calculation of flow coefficient based on the standard ISA 75.01 and IEC 60534 was performed as follows $[2,3]$ :

$C_{V}=\frac{w}{27.3 \times \sqrt{\rho \times \Delta P}}$

,where $\mathrm{w}$ is the mass flow rate in $\mathrm{kg} / \mathrm{h}, \rho$ is fluid density in $\mathrm{kg} / \mathrm{m} 3$, and $\Delta P$ stands for the pressure drop across the valve in 
bar. The equal percentage theoretical characteristic of valve is calculated from the formula of equation (2). [7]

$q=a^{1-h}$

, where

$q=\frac{C V \text { at current opening }}{C V \text { at fully-open }(\text { Rated } C V)}$

$a=\frac{C V \text { at Minimum opening }}{C V \text { at fully-open }(\text { Rated } C V)}=0.03$

$h=$ Travel Percentage $=\frac{\text { Plug Distance From Seat }(\text { Travel })}{\text { Travel at fully-open }(\text { Stroke })}$

(5)

The resulting curve was compared with the ideal equal percentage curve based on the criteria suggested by ANSI/ISA75.11.01-1985 (R2002) [9] which provides limits for acceptable deviations of actual inherent characteristic from the characteristic reported by manufacturer. (Figure 6)

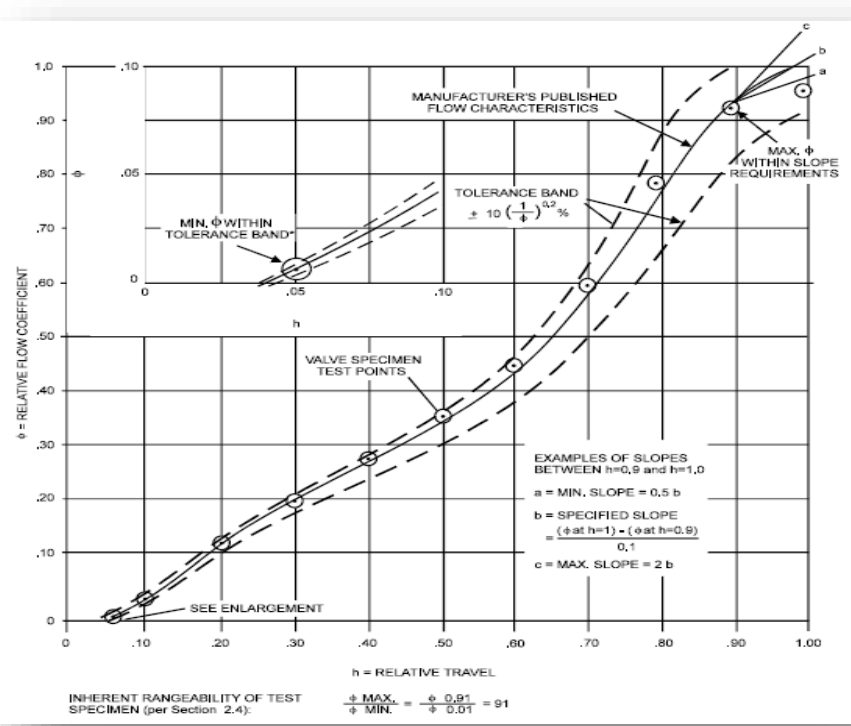

Figure 6- Acceptable range of deviations from catalogue curve by ANSI/ISA75.11.01-1985 (R2002) [9]

\section{3- RESULTS}

The obtained results - in terms of valve coefficient $(\mathrm{CV})$ versus travel percentage of valve stem - from the first scenarios (Fully-open to Fully-closed) are shown and compared to the theoretical equal percentage characteristic from equation (3) in Figure 7. As it was expected, the equal percentage characteristic was properly followed by the performance curve of the trim. However, there seemed to be minor deviations around the opening of $80 \%$.
Additionally, the results of the other two scenarios were demonstrated in Fig. 8. It seems that the cyclic hysteresis effect is more considerable in the range $60 \%-80 \%$ opening where it caused fluctuations and minor discrepancy in the results of the three scenarios. $\mathrm{R}^{2}$ value from statistical analyses of six pairs of results are shown in Table 2. Based on the values, the first scenario is the closest to the ideal characteristic and the closing cycles (scenarios $1 \& 3$ ) seem to be more similar in pattern than the other pairs.

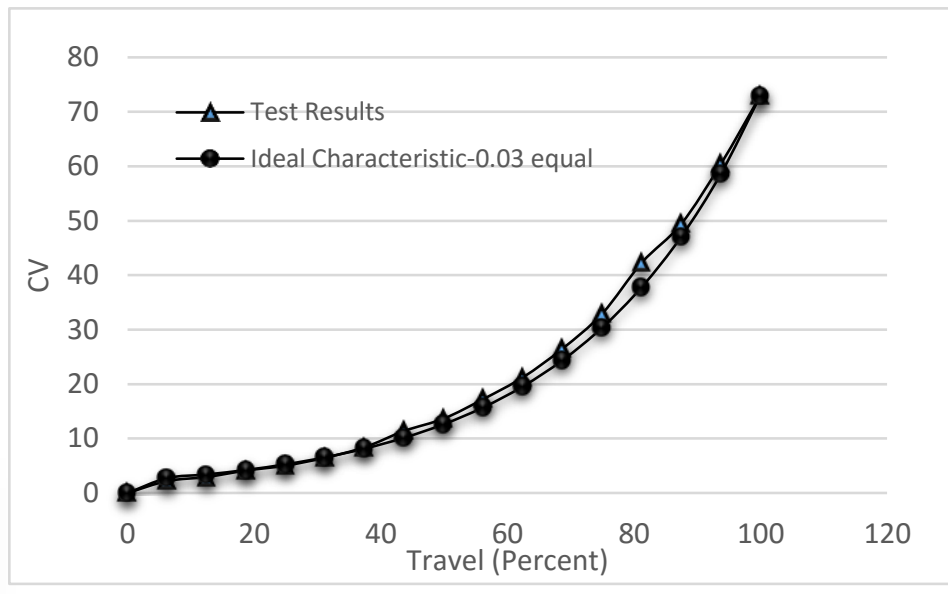

Figure 7- Flow characteristic obtained from the test vs. the ideal equal percentage flow characteristic

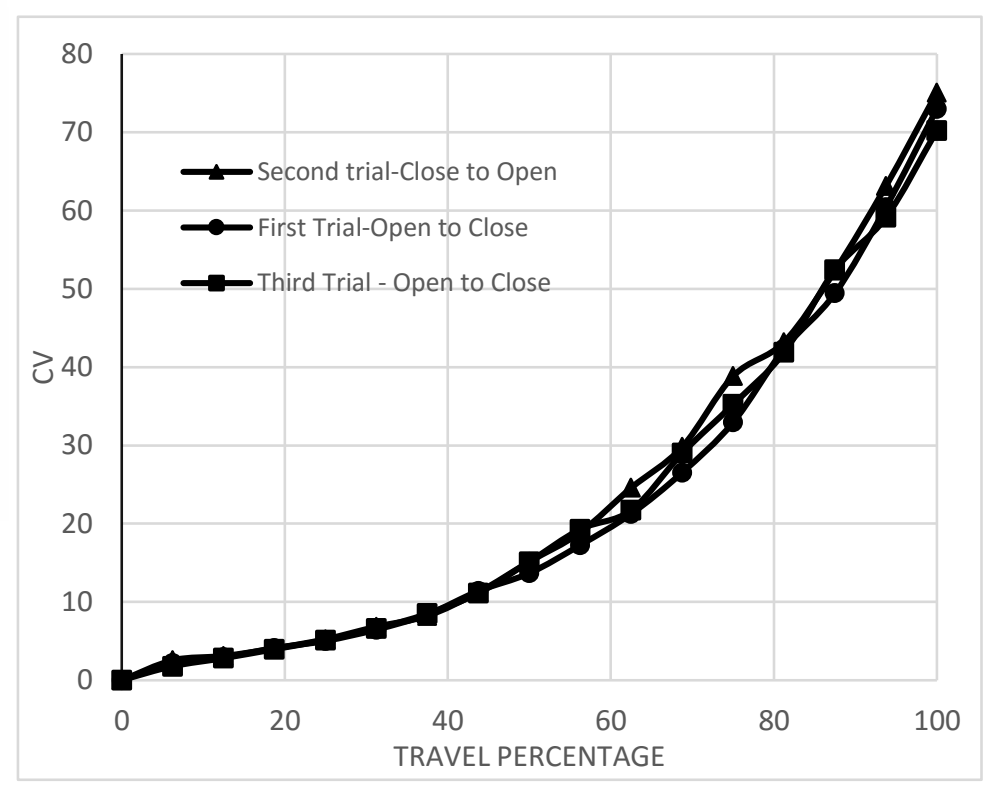

Figure 8- Flow characteristic obtained from the test vs. the ideal equal percentage flow 
Table 2- R2 values for trend lines of the three test scenarios with theoretical curve. Ideal characteristic is considered equal percentage with Min CV of 3\%

\begin{tabular}{|c|c|c|c|c|c|}
\hline $\begin{array}{c}\text { Ideal- } \\
\text { Test1 }\end{array}$ & $\begin{array}{c}\text { Ideal- } \\
\text { Test2 }\end{array}$ & $\begin{array}{c}\text { Ideal- } \\
\text { Test3 }\end{array}$ & $\begin{array}{c}\text { Test1- } \\
\text { Test2 }\end{array}$ & $\begin{array}{c}\text { Test1- } \\
\text { Test3 }\end{array}$ & $\begin{array}{c}\text { Test2- } \\
\text { Test3 }\end{array}$ \\
\hline 0.991 & 0.963 & 0.975 & 0.985 & 0.990 & 0.984 \\
\hline
\end{tabular}

\section{4- DISCUSSION OF RESULTS}

The three recorded equal percentage characteristic curves are shown and compared for hysteresis of the hydraulic system denote fluctuations in the measurements at some points along the valve full stroke. The data of the first scenario (fully-open to fully closed condition) are compared to the ideal equal percentage characteristic of reference [7] shown in Equation (2). This is displayed in Figure 7 where the deviations from ideal equal percentage characteristic are almost negligible for the first scenario. However, the deviation becomes more noticeable in the two remaining repetitions of the scenario (scenarios 2 and 3). This can be attributed partly to measurement and reading errors.

The comparison of the trend of test results in terms of Rsquared in Table 2 shows that the test results are acceptable valid regression models of the theoretical data. However, the similarity of the trend line is more noticeable in opening curves (higher R-squared value for test1-test 3 comparison). For better evaluation of the quantitative validity of test results, a statistical T-test was performed. (Table 3) In this table, the average of the three values for each test point in terms of $\mathrm{CV}$ has been considered as test results, which is compared to the ideal characteristic. From equation (6), the result of the T-test show that the averages of the two sets of data do not differ significantly. As it can be seen, the statistical analysis including the qualitative $\left(\mathrm{R}^{2}\right)$ and quantitative (T-test) results demonstrate acceptable results.

Table 3- T-Test comparison for ideal characteristic and test data

\begin{tabular}{|l|l|l|}
\hline \multicolumn{1}{|c|}{ Parameter } & \multicolumn{1}{|c|}{ Ideal } & \multicolumn{1}{c|}{ Test Result } \\
\hline Mean & 21.16 & 22.807055 \\
Variance & 461.74 & 500.219218 \\
Observations & 17 & 17 \\
t Stat & 0.21 & \\
P(T<=t) one-tail & 0.41 & \\
t Critical one-tail & 1.69 & \\
P(T<=t) two-tail & 0.82 & \\
t Critical two-tail & 2.03 & \\
\hline
\end{tabular}

,the requirement of the T-test acceptability is as follows:

$$
\begin{gathered}
-\mathrm{t} \text { Critical two }- \text { tail } \leq \mathrm{t} \text { Stat } \leq+\mathrm{t} \text { Critical two }- \text { tail } \\
-2.03 \leq 0.2 \leq+2.03
\end{gathered}
$$

More precise analysis can be made through the method of ANSI/ISA-75.11.01-1985 (R2002) which provides limits for acceptable deviations of actual inherent characteristic from the characteristic reported by manufacturer. The Ensemble CV in Equation (7) at fully-open condition is average for the three scenarios at this condition. As it is observed, the maximum relative error is $4.3 \%$ at fully-open condition for the third scenario where the calculated CV is 75.09 while the nominal $\mathrm{CV}$ equals 72. In addition, the changes of relative error threshold from reference [9] is compared to the actual relative error from ideal equal percentage trim (the average of three scenarios at each control point has been used) in Figure 9. It can be inferred from the Figure that, although the average values of CV do not exceed the threshold level, the values of CV tend to slightly fall beyond the levels at some points of the threefold scenarios. The highest relative errors, which are accompanied by slightly exceeding the threshold, occur when the valve stem is $6.25 \%, 43.75 \%$ and $81.25 \%$ open.

$$
\text { Average Ensemble CV at full Travel }=\frac{73+70.23+75.09}{3}=72.77
$$

Nominal Rated $C V=72$

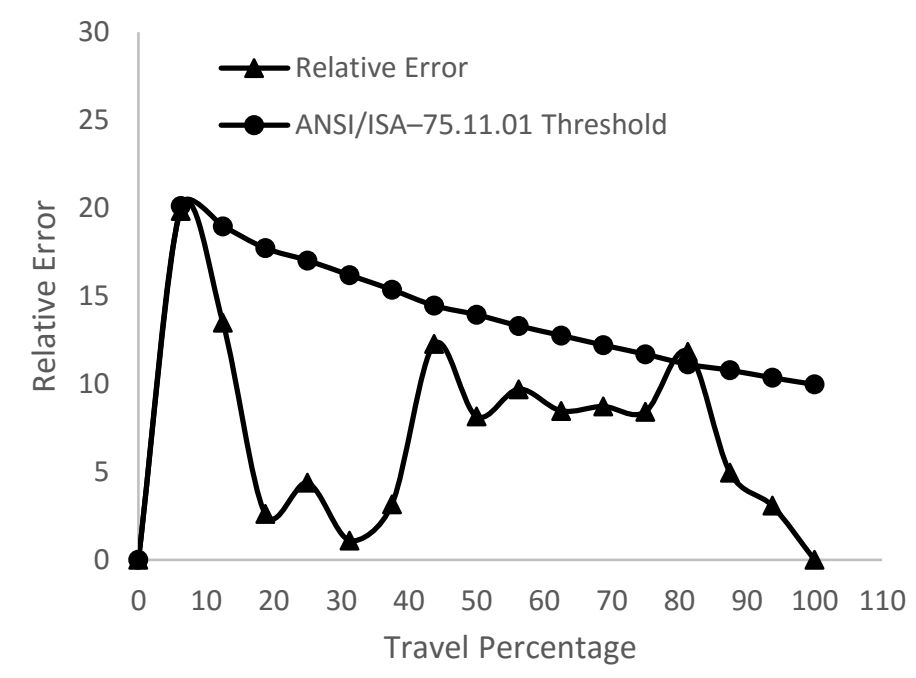

Figure 9- Comparison between experiment relative error (average of three scenarios at each control point has been used) and allowable limit of error from [9]

The compound error of CV calculation from the measured parameters ( $w$ and pressure drop) is presented in Equation (8),

$\delta(C V)=\left[\frac{1}{27.3 \times \sqrt{\rho \times \Delta P}} \times \delta(w)\right]+\left[\frac{w}{27.3 \times 2 * \times \sqrt{\rho \times \Delta P} \times \Delta P} \times \delta(\Delta P)\right]$

,where $\delta(C V)$ is the ensemble error of $\mathrm{CV}, \delta w$ error of flow measurement which is $2 \%$ for based on the specifications of the measurement device, and $\delta(\Delta P)$ represents the error of pressure drop measurements which equals two times the error 
of one reading (totally $4 \%$ since the pressures are read at input and output and then they are subtracted).

As it can be inferred from Figure 10, the measurement compound error grows as the calculated $\mathrm{CV}$ is increased. This occurs due to higher contribution of flow (than pressure drop) to $\mathrm{CV}$ measurements in control valve. As a result of the proportionality of flow and $\mathrm{CV}$, and given the somewhat constant pressure drop throughout the test, the width of the error bars grow exponentially similar to the equal percentage characteristic of valve. By adding the values of measurement compound relative error to the relative errors of the experiment results from ideal characteristic (Figure 9), the threshold is surpassed only at $81.5 \%$ travel by the amount of $3 \%$ relative error which can be considered negligible.

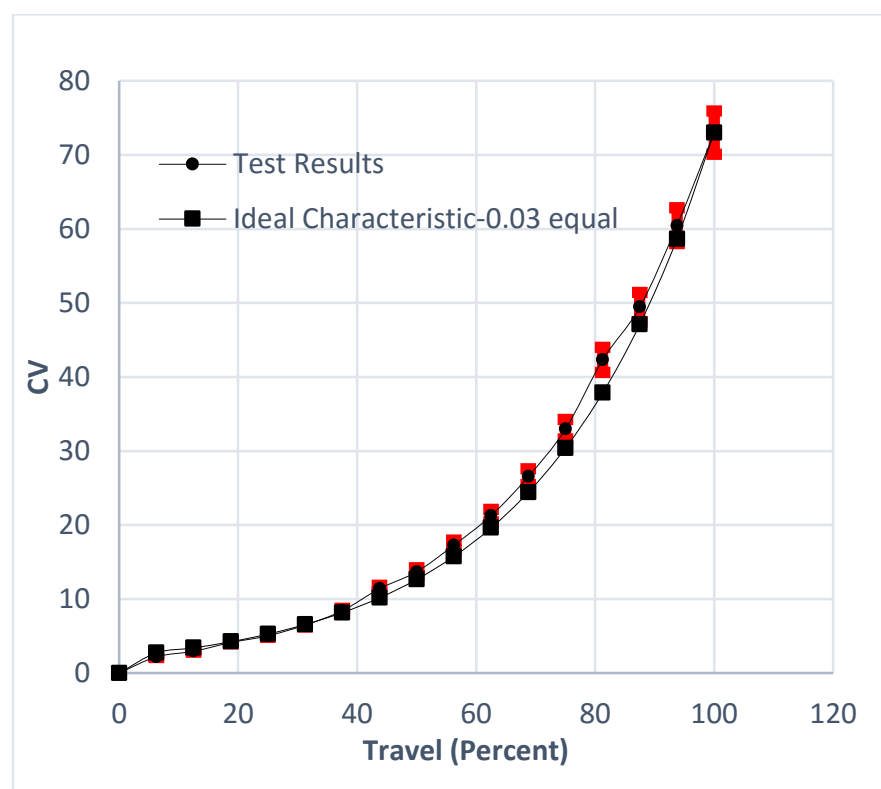

Figure 10- Compound error of CV measurement based on the accuracy of measurement devices shown for the results of the first scenario (fully-open to fully-closed) compared with exact ideal equal percentage characteristic (with minimum relative $\mathrm{CV}$ of 0.03 )

\section{5- CONCLUSIONS}

A 3 in. globe control valve with top guided contoured trim was tested experimentally for its performance curve on a standard 3 in. control loop set-up. The performance test was repeated three times starting with a fully-open to fully-closed condition of valve and the data of water flow and pressure drop across the valve were recorded after each $1 \mathrm{~mA}$ change in positioner input signal (equivalent to $6.25 \%$ change in valve stem travel after calibration of positioner).

The tests were carried out in three various scenarios including successive opening and closing cycles. The results show negligible hysteresis existing between the cycles with opposite moving directions of valve stem. The valve characteristic was compared to the ideal characteristic of reference [7] which demonstrated acceptable results not exceeding the threshold of relative error addressed by reference [9]. However, it was later shown that the compound measurement error of $\mathrm{CV}$ can push the values slightly beyond the acceptable limits of reference [9]. Although, the overall results of the experiment are acceptable, In order to further reduce the compound errors of measurements, there are a number of methods including use of a pressure subtractor measurement device and performing sensitivity analysis.

\section{6- ACKNOWLEDGEMENTS}

The authors would like to express their appreciations to Rasta Group Industrial Valve Co. for financially supporting the project. Instrumentation specialists $\mathrm{Mr}$. Zargaran and $\mathrm{Mr}$. Arbabi for accompanying the project to the very end. In addition, the cooperation of the Iranian Ministry of Power is appreciated for making the necessary arrangements for valve set-up and installation.

\section{7- REFERENCES}

[1] "Valve Sizing." Process Automation Handbook: 133-41. doi:10.1007/9781-84628-282-9_20.

[2] "Industrial-process control valves. Flow capacity." doi:10.3403/02604354.

[3] "Industrial-process control valves." doi:10.3403/30178780.

[4] ANSI/ISA-75.02.01-2008 Control Valve Capacity Test Procedures

[5] IEC 60534-2-3 (1997): Industrial-Process Control Valves, Part 2: Flow Capacity, Section 3: Test Procedures

[6] Vikas L. Karade, Ambadas B. Shinde, Praveen V. Pol (2015), 'Control Valve Coefficient Testing', IJIRT | Volume 2 Issue 6 | ISSN: 2349-6002

[7] Borden, Guy, and Paul G. Friedmann. Control valves. Research Triangle Park, NC: Instrument Society of America, 1998.

[8] Aragon-Camarasa, G., G. Aragon-Gonzalez, A. Canales-Palma, and A. Leon-Galicia. "Test Bench for Process Control Valves." Electronics, Robotics and Automotive Mechanics Conference (CERMA 2007), 2007. doi:10.1109/cerma.2007.4367675

[9] Inherent flow characteristic and rangeability of control valves. Research Triangle Park, NC: Instrument Society of America, 1984. 